\title{
Liver transplantation for small hepatocellular carcinoma
}

\author{
Naoko Kamo, Toshimi Kaido, Shintaro Yagi, Hideaki Okajima, Shinji Uemoto \\ Division of Hepato-Biliary-Pancreatic and Transplant Surgery, Department of Surgery, Graduate School of Medicine, Kyoto University, Kyoto, Japan \\ Contributions: (I) Conception and design: N Kamo, T Kaido; (II) Administrative support: All authors; (III) Provision of study materials or patients: All \\ authors; (IV) Collection and assembly of data: N Kamo, T Kaido; (V) Data analysis and interpretation: N Kamo, T Kaido; (VI) Manuscript writing: \\ All authors; (VII) Final approval of manuscript: All authors. \\ Correspondence to: Toshimi Kaido, MD, PhD. Division of Hepato-Biliary-Pancreatic and Transplant Surgery, Department of Surgery, Graduate \\ School of Medicine, Kyoto University, 54 Kawahara-cho, Shogoin, Sakyo-ku, Kyoto 606-8507, Japan. Email: kaido@kuhp.kyoto-u.ac.jp.
}

Background: We treat small hepatocellular carcinoma (HCC) $\leq 3 \mathrm{~cm}$ in diameter by liver transplantation (LT) considering liver reserve and HCC localization, and when other treatment would be ineffective. However, the outcomes of LT and the clinicopathological features of small HCC $\leq 3 \mathrm{~cm}$ in diameter are not clear. We analyzed the outcomes of LT for small HCC $\leq 3 \mathrm{~cm}$ in diameter.

Methods: Between February 1999 and August 2015, 223 patients underwent LT for HCC at Kyoto University Hospital. We analyzed the proportion of small HCC $\leq 3 \mathrm{~cm}$ in diameter (small HCC) among all patients, the background of small HCC, survival and recurrence rates within and beyond the Milan criteria (MC), Kyoto criteria (KC) $[\leq 5 \mathrm{~cm}, \mathrm{~N} \leq 10$, des-gamma-carboxy prothrombin $(\mathrm{DCP}) \leq 400]$, and rates of survival and recurrence after LT with or without pretreatment.

Results: Among the 223 patients, 159 (71\%) had small HCC accompanied by hepatitis B virus (HBV), 43 (27\%); hepatitis C virus (HCV), 96 (61\%); HBV, HCV, 5 (3\%) and non B non C, 15 (9\%). One hundred and fourteen $(72 \%$ ) patients were male with a tumor radius of 2 (range, 0.4-3) cm; number of tumors, 2 (range, 1-186); alpha-fetoprotein (AFP) 28.5 (range, 1.3-12,727) and DCP 42 (range, 5-20,600). The tumors were well, moderately and poorly differentiated in 22 (14\%), 105 (66\%) and 24 (15\%) patients, respectively. Among the patients, 124 (78\%) and 132 (83\%) were within the MC and KC, respectively. One-, threeand five-year survival rates associated with tumors within and beyond the MC were $87 \%, 81 \%$ and $79 \%$ vs. $94 \%, 76 \%$, and $70 \%$, respectively $(\mathrm{P}=0.430)$ and recurrence rates were significantly lower in patients within $\mathrm{MC}$ than in patients beyond $\mathrm{MC}(\mathrm{P}<0.001)$. One-, three- and five-year survival rates associated with tumors within and beyond $\mathrm{KC}$ were $89 \%, 85 \%$, and $83 \%$ vs. $89 \%, 58 \%$, and $50 \%$, respectively $(\mathrm{P}<0.001)$ and recurrence rates were $2 \%, 3 \%$, and $4 \%$ vs. $21 \%, 37 \%$, and $47 \%$, respectively $(\mathrm{P}<0.001)$. Survival and recurrence rates were significantly higher and lower, respectively, among patients whose tumors were within, than beyond the KC. The one, three and five-year survival rates of patients with or without pretreatment were $89 \%, 79 \%$, and $75 \%$ vs. $86 \%, 81 \%$, and $81 \%$, respectively $(\mathrm{P}=0.315)$ and recurrence rates were $6 \%$, $10 \%$, and $15 \%$ vs. $2 \%, 2 \%$, and $2 \%$, respectively $(\mathrm{P}=0.040)$. Recurrence rates were significantly higher in patients with pretreatment than in those without pretreatment.

Conclusions: Outcomes after LT were favorable for patients with small HCC that meet MC criteria or are less biologically malignant.

Keywords: Small hepatocellular carcinoma (HCC); outcome; transplantation

Submitted Mar 01, 2016. Accepted for publication Apr 07, 2016.

doi: $10.21037 /$ hbsn.2016.05.03

View this article at: http://dx.doi.org/10.21037/hbsn.2016.05.03 


\section{Introduction}

Hepatocellular carcinoma (HCC) is the most common type of hepatic malignancy and the second most common cause of cancer-related mortality worldwide (1). During the past few decades, therapeutic options for HCC have increased and now include hepatic resection (HR), liver transplantation (LT), radiofrequency ablation (RFA), and transcatheter arterial chemoembolization (TACE). The choice of treatment modality depends on the size, number and location of tumors, anatomical considerations and liver function.

The Barcelona Clinic Liver Cancer algorithm is the most widely applied staging system. Patients with single liver tumors or as many as three nodules $<3 \mathrm{~cm}$ are classified as having very early or early-stage cancer, and benefit from resection, transplantation, or ablation (2). HR has long been considered the main treatment option for HCC, but high recurrence rates after HR impact the prognosis and survival of patients. Thus, clarifying which factors are involved in survival and in tumor recurrence after HR is crucial. In contrast, LT has evolved to become an important surgical strategy for patients with HCC. This procedure has also become an established treatment for end-stage liver disease (3).

Regardless of tumor size, the Japanese treatment algorithm for HCC proposed HR for Child-Pugh A and B liver function (4). In contrast, LT is recommended for patients with Child-Pugh $\mathrm{C}$ liver function within the Milan criteria (MC). However, indications for HR and LT vary among institutions. Many transplant centers around the world use expanded transplantation criteria for HCC beyond MC (5-10). LT is sometimes applied to patients with Child-Pugh A and B liver function who cannot undergo HR or RFA due to liver dysfunction or tumor location.

Although outcomes of LT for HCC (5-11) have been reported, little is known about those of LT and clinicopathological features for small $\mathrm{HCC} \leq 3 \mathrm{~cm}$ in diameter (small HCC). Here, we retrospectively analyzed the impact of LT for small HCC upon long-term survival and recurrence.

\section{Methods}

We retrospectively analyzed data from 223 patients who underwent LT for HCC at Kyoto University Hospital between February 1999 and August 2015. The database was designed for patients with small HCC. We excluded patients who were preoperatively diagnosed with HCC accompanied by vascular invasion or distant metastasis based on findings of computed tomography (CT) and magnetic resonance imaging (MRI), and whose data regarding items such as alpha-fetoprotein (AFP) and DCP values, exact preoperative tumor size or number of tumors were missing. The Ethics Committee of Kyoto University approved the study, which proceeded in accordance with the Declaration of Helsinki, 1996.

Standard selection criteria for LT recipients and the surgical techniques for both donor and recipient operations have been described in detail (12-14). Graft and remnant liver volumes in donors were preoperatively estimated using three-dimensional reconstructed images of the hepatic vascular anatomy produced using a software package based on reconstructed, multi-detector CT images of the liver. Donor candidates were finally selected and approved by our institutional living donor (LD) LT committee. Until December 2006, we used primary institutional selection criteria for LDLT for HCC, which included any size or number of tumors; however, HCC had to have been free of distant metastasis or gross vascular involvement at the time of preoperative imaging. Since January 2007, we have applied the Kyoto criteria (KC) as described (10). Briefly, risk factors for recurrence were analyzed in 136 patients who underwent LT for HCC between February 1999 and December 2006. Based on the results of a multivariate analysis, we established the $\mathrm{KC}$ consisting of three independent significant risk factors for recurrence: tumor number $\leq 10$, maximal diameter of each tumor $\leq 5 \mathrm{~cm}$ and serum DCP levels $\leq 400 \mathrm{mAU} / \mathrm{mL}$. The absence of vitamin $\mathrm{K}$ or antagonist II induces production of the protein, DCP, which is a tumor marker of HCC. Serum levels of DCP significantly correlate with a poor prognosis (15-19). Until January 2011, we used the standard immunosuppression protocol consisted of tacrolimus and low-dose steroid $(20,21)$. After February 2011, we employed steroidfree protocol using mycophenolate mofetil as described elsewhere excluding the cases with ABO-incompatible transplantation or with steroid administration before LT (22). None of the patients were switched to mammalian target of rapamycin inhibitor.

Age, sex, tumor radius, number, AFP, DCP, pathological classification, etiology, model for end stage liver disease (MELD) scores were analyzed for each patient. Overall survival and recurrence rates after LT within and beyond the MC, KC, and with or without treatment before LT 
Table 1 Clinicopathological characteristics of patients

\begin{tabular}{|c|c|}
\hline Characteristics & $n=159$ \\
\hline Age (years) & 57 [27-69] \\
\hline Sex (male/female) & $114 / 45$ \\
\hline \multicolumn{2}{|l|}{ Etiology } \\
\hline Viral hepatitis C & 96 \\
\hline Viral hepatitis B & 43 \\
\hline Viral hepatitis C/B & 5 \\
\hline Others & 15 \\
\hline AFP & $28.5(1.3-12,727)$ \\
\hline DCP & $42[5-20,600]$ \\
\hline Child Pugh (A/B/C) & $20 / 59 / 80$ \\
\hline MELD & $15.8[4-41]$ \\
\hline Maximum tumor size (cm) & $2[0.4-3]$ \\
\hline Classification & Well $23 / \bmod 112 /$ poor 24 \\
\hline Number of tumors & $2[1-186]$ \\
\hline Preoperative treatment & $109(69 \%)$ \\
\hline HR & 1 \\
\hline $\mathrm{HR}+\mathrm{TACE}$ & 4 \\
\hline $\mathrm{HR}+\mathrm{RFA}$ & 3 \\
\hline $\mathrm{HR}+\mathrm{TACE}+\mathrm{RFA}$ & 7 \\
\hline TACE & 33 \\
\hline RFA & 22 \\
\hline TACE + RFA & 39 \\
\hline Radiation & 0 \\
\hline Chemotherapy & 0 \\
\hline Milan criteria met & $124(78 \%)$ \\
\hline Kyoto criteria met & $132(83 \%)$ \\
\hline
\end{tabular}

AFP, alpha-fetoprotein; DCP, des-gamma-carboxy prothrombin; MELD, model for end-stage liver disease; HR, hepatic resection; TACE, transcatheter arterial chemoembolization; RFA, radiofrequency ablation.

were also analyzed. Continuous variables are expressed as medians and ranges. Patients characteristics between groups were compared using $\chi^{2}$ test for multinomial categorical variables and paired $t$-test for continuous variables. Cumulative overall survival and recurrence rates were calculated using the Kaplan-Meier method, and differences between curves were evaluated using the log-rank test. Values with $\mathrm{P}<0.05$ were considered significant. All data were statistically analyzed using SPSS computer software (SPSS Inc., Chicago, IL, USA).

\section{Results}

Among the 223 patients, 159 (71\%) had small HCC. In addition to having small HCC, 96 (61\%) and 43 (27\%) patients were also positive for hepatitis $\mathrm{C}$ virus (HCV) antibody and hepatitis B surface antigen (HBsAg), respectively. Five (3\%) and 15 (9\%) also had both $\mathrm{HCV}$ antibody and HBsAg, and neither, respectively. The median age was 57 (range, 27-69) years. Table 1 describes the clinicopathological characteristics of patients with small HCC. LDs provided the livers for all LT for these HCC. The number of recipients beyond MC, but within $\mathrm{KC}$ or beyond $\mathrm{KC}$ are 21, 14 patients, respectively. On the other hand, the number of recipients within $\mathrm{MC}$ and within $\mathrm{KC}$ or beyond $\mathrm{KC}$ are 111, 13 patients, respectively.

We analyzed the overall survival rates of patients after LDLT for HCC $\leq 3 \mathrm{~cm}$ in diameter between February 1999 and August 2015. One-, three-, and five-year overall survival rates within and beyond the MC were $87 \%, 81 \%$, and $79 \%$ vs. $94 \%, 76 \%$, and $70 \%$, respectively and did not significantly differ $(\mathrm{P}=0.430$; Figure $1 A)$. In contrast, one-, three- and five-year recurrence rates were significantly lower within $(2 \%, 4 \%$, and $6 \%$, respectively) than beyond $(15 \%, 21 \%$, and $28 \%$, respectively) the $\mathrm{MC}(\mathrm{P}<0.001$, Figure 1B).

The one-, three- and five-year overall survival rates for patients within and beyond the $\mathrm{KC}$ were $89 \%, 85 \%$, and $83 \%$ vs. $89 \%, 58 \%$, and $50 \%$, respectively. Overall survival rates were significantly higher for patients within, than beyond the $\mathrm{KC}(\mathrm{P}<0.001$, Figure $2 A)$. The one-, three- and five-year recurrence rates within and beyond the $\mathrm{KC}$ were $2 \%, 3 \%$, and $4 \%$ vs. $21 \%, 37 \%$, and $47 \%$, respectively. Recurrence rates were significantly lower for patients within, than beyond the $\mathrm{KC}(\mathrm{P}<0.001$; Figure $2 B)$.

We analyzed overall survival and recurrence rates according to whether or not the patients had been treated before LT with HR, TACE, RFA, or percutaneous ethanol injection therapy (PEIT) to determine their impact upon small HCC. Among the patients, 109 (69\%) of 159 were treated before LT. Fifteen patients had HR and local therapy including TACE, RFA and PEIT. Among these, one was treated only with HR. In contrast, 94 patients had local 


\section{A}

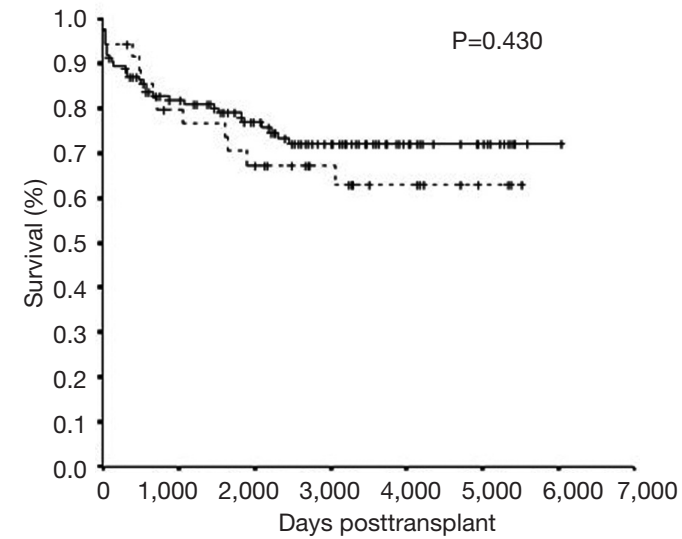

B

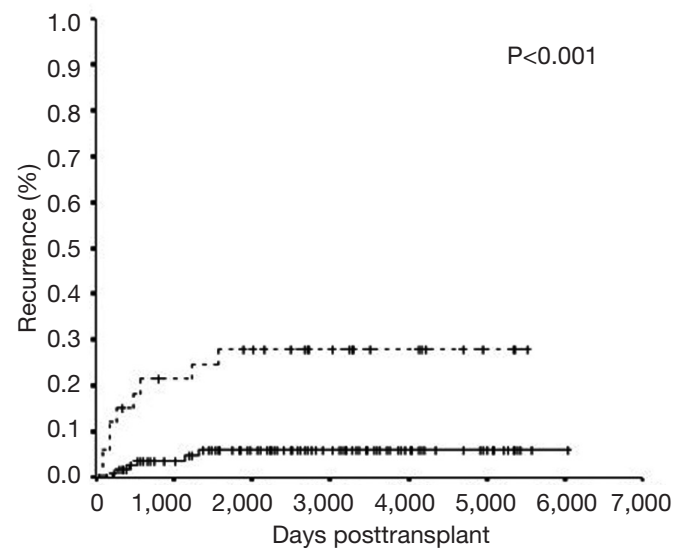

Figure 1 Overall survival (A) and recurrence (B) rates of LT for small HCC within and beyond MC. LT, liver transplantation; HCC, hepatocellular carcinoma; MC, Milan criteria.

\section{A}
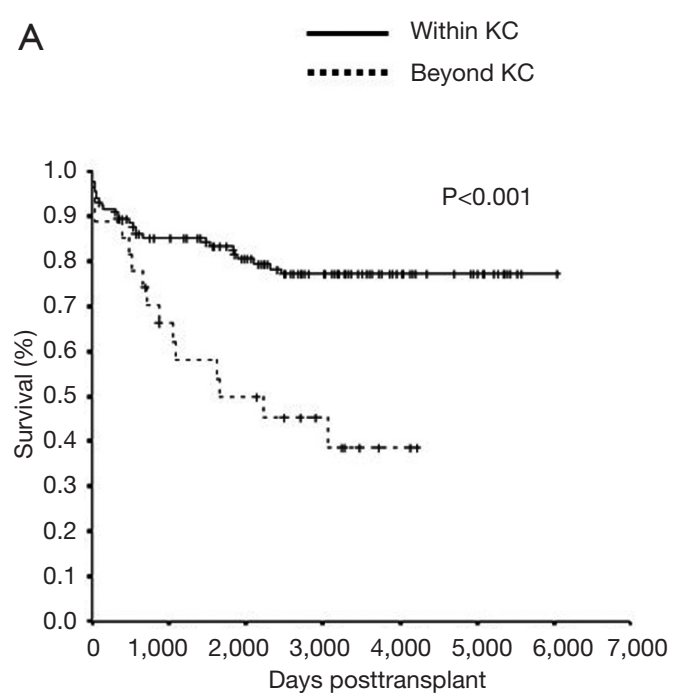

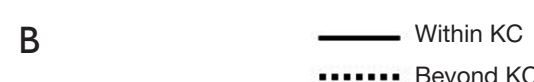

...... Beyond KC

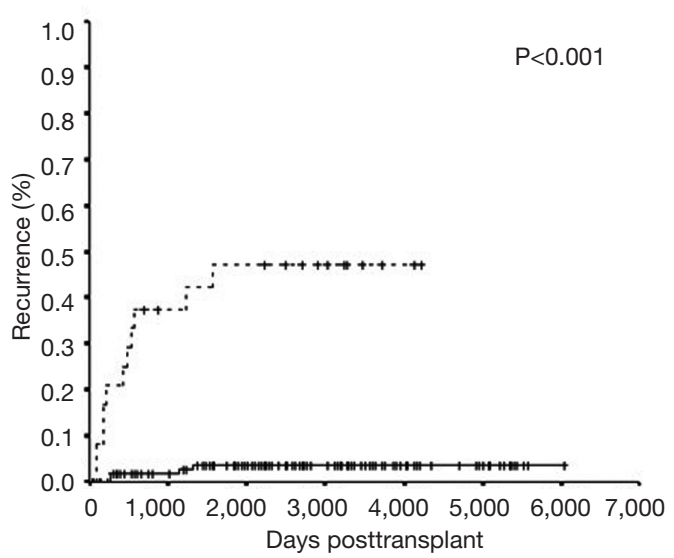

Figure 2 Overall survival (A) and recurrence (B) rates of LT for small HCC within and beyond KC. LT, liver transplantation; HCC, hepatocellular carcinoma; KC, Kyoto criteria.

therapy before LT. Table 2 shows the clinicopathological characteristics of patients with or without preoperative treatment. MELD score and the ratio within the MC were significantly higher in patients without preoperative treatment. The one-, three- and five-year overall survival rates in the groups with or without treatment before LT were $89 \%, 79 \%$, and $75 \%$ vs. $86 \%, 81 \%$, and $81 \%$, respectively (no significant difference; $\mathrm{P}=0.315$; Figure $3 A$ ). By contrast, one-, three- and five-year recurrence rates were $6 \%, 10 \%$, and $15 \%$ vs. $2 \%, 2 \%$, and $2 \%$, respectively. The recurrence rates were significantly lower in patients without, than with treatment before LT $(\mathrm{P}=0.040$; Figure $3 B)$.

Next, to show the consistency between preoperative radiologic findings and the pathological number and tumor 
Table 2 Clinicopathological characteristics of patients between with and without preoperative treatment

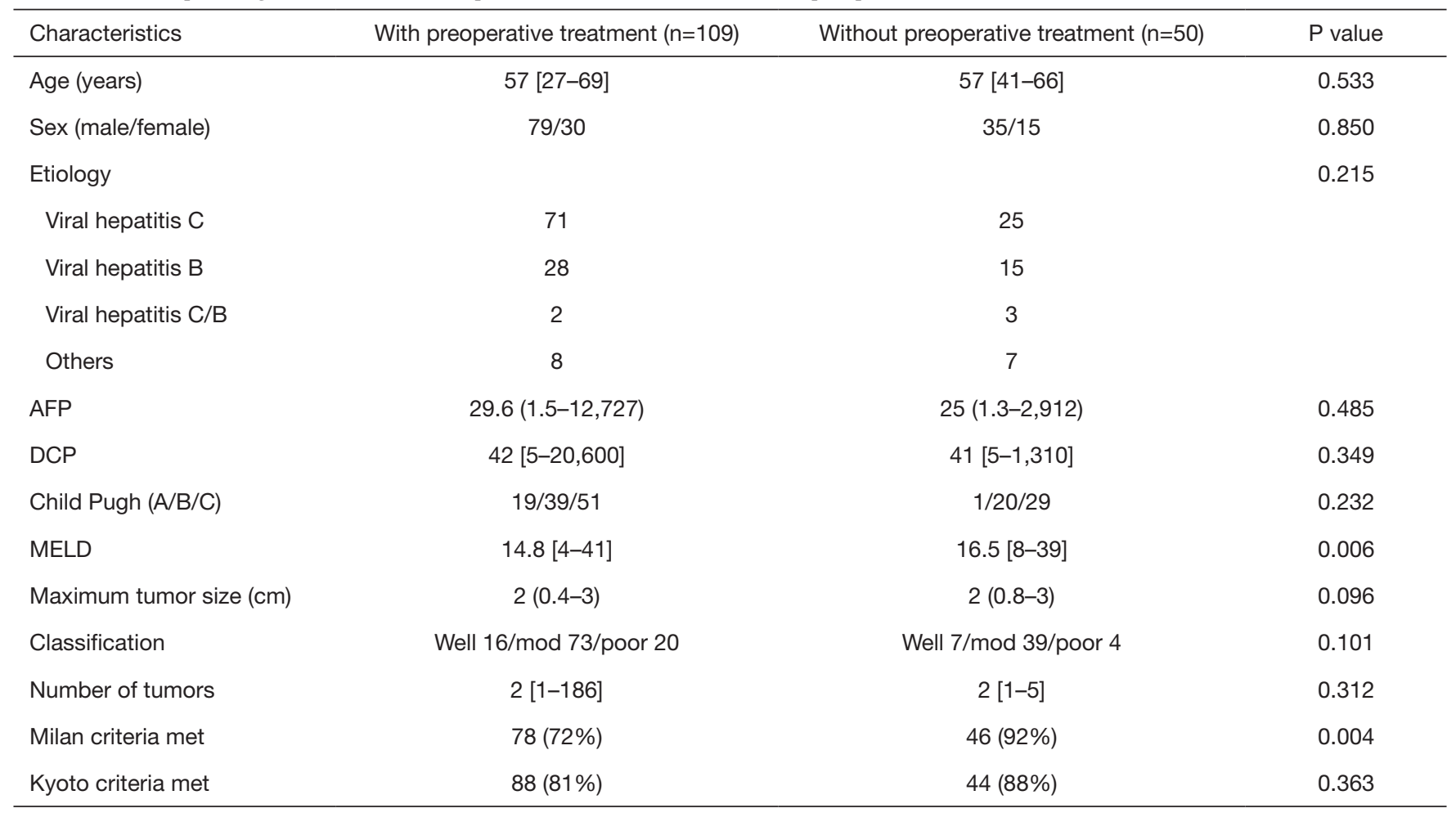

AFP, alpha-fetoprotein; DCP, des-gamma-carboxy prothrombin; MELD, model for end-stage liver disease.
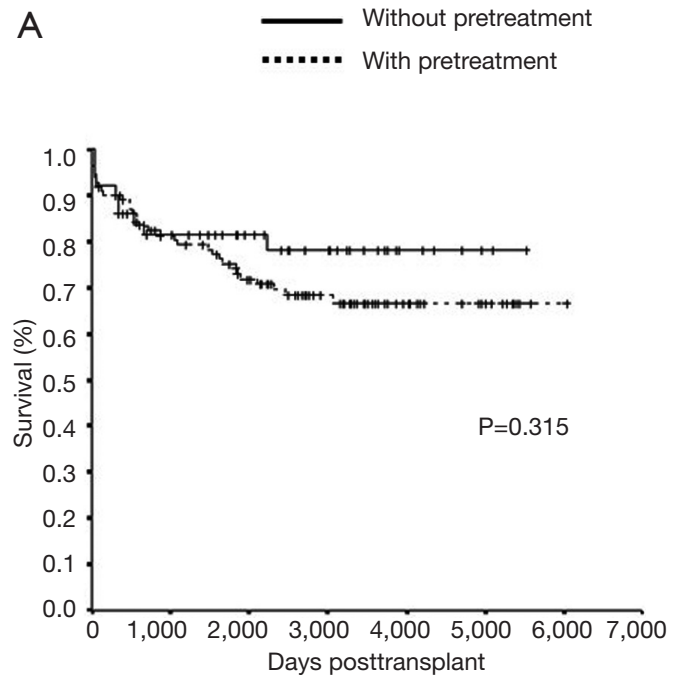

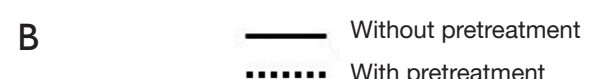

...... With pretreatment

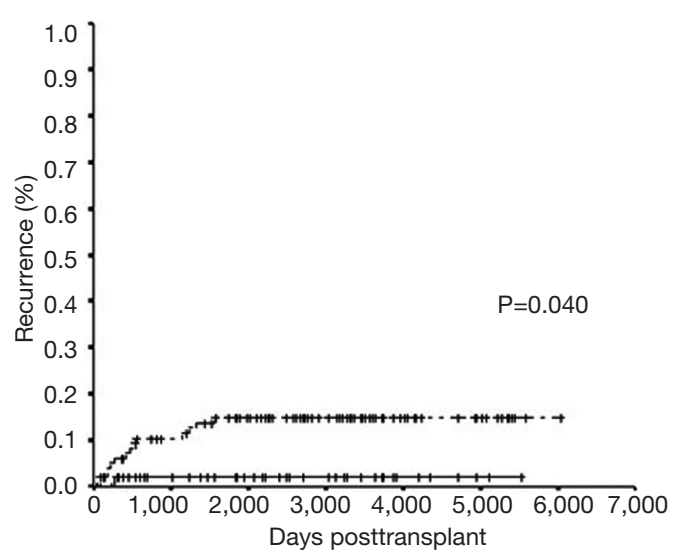

Figure 3 Overall survival (A) and recurrence (B) rates of LT for small HCC with or without pretreatment. LT, liver transplantation; HCC, hepatocellular carcinoma. 
Table 3 Evaluations about the consistency between pre- and postoperative diagnosis

\begin{tabular}{llc}
\hline Preoperative diagnosis & Postoperative diagnosis & $\mathrm{n}=159$ \\
\hline Within MC & Beyond MC & $24(19 \%)$ \\
Within MC & Within MC & 100 \\
Beyond MC & Within MC & $6(17 \%)$ \\
Beyond MC & Beyond MC & 29 \\
\hline
\end{tabular}

MC, Milan criteria.

size of explant liver, we evaluated the consistency between pre- and postoperative diagnosis (Table 3). Twenty-four of $124(19 \%)$ patients who were preoperatively diagnosed within the MC were underestimated. On the other hand, 6 of $35(17 \%)$ patients who were preoperatively diagnosed beyond the $\mathrm{MC}$ were overestimated.

\section{Discussion}

The prognosis of patients with HCC has significantly improved over the past three decades due to advances in treatment for HCC. The choice of treatment modality for HCC depends on the size, number and location of tumors, anatomical considerations, and liver function. Generally speaking, LT is recommended for patients with Child-Pugh C liver function within the MC. Patients with Child-Pugh A or B liver function and uncontrolled HCC were referred to our hospital for LT as second-line treatment. As a result, most patients with small HCC who had relatively preserved liver function underwent LT. In fact, 20 and 59 patients with Child-Pugh A and B liver function, respectively, were included in this study.

Several transplant centers around the world use expanded transplantation criteria for HCC beyond the MC (5-10). Here, application of the $\mathrm{KC}$ resulted in favorable outcomes for patients undergoing LDLT for small HCC between February 1999 and August 2015. The one-, three- and five-year overall survival and recurrence rates for patients within the KC were $89 \%, 85 \%, 83 \%$ and $2 \%, 3 \%, 4 \%$, respectively, with significant differences between within and beyond the $\mathrm{KC}$. The important issue of the $\mathrm{KC}$ is that it includes DCP, a marker of tumor aggressiveness that reflects biological malignancy. Our findings showed that the KC can effectively exclude patients with a high risk for recurrence before undergoing LT. In the present study, 21
(13.2\%) of 159 patients beyond the Milan, but within the KC were supported by the expanded criteria.

We previously reported that repeated non-transplant treatment for recurrent HCC before LDLT might increase risk of recurrence and impair the survival advantages conferred by LDLT (21). However, LDLT might offer the chance of a cure for patients with uncontrolled disease (21). We also previously found that treatment for HCC before LT is an independent risk factor for recurrence (11). Taking this into consideration, the present outcomes of patients with small HCC seem to support our previous findings. Recurrence rates in the present study were significantly higher among patients who were treated before LT compared with those who were not.

Others have shown that recurrence rates of HCC are significantly lower after deceased donor (DD) LT than after LDLT $(23,24)$. These studies found adequate time to assess the biological behavior of tumors, and could exclude patients with a high risk of recurrence before undergoing LT. Moreover, differences in surgical techniques or liver regeneration between LDLT and DDLT might influence rates of tumor recurrence (25). However, none of our patients underwent DDLT for small HCC and further investigation of more patients is required in the future.

Some limitations of this study must be considered. The numbers of recipients within and beyond the MC, within and beyond the $\mathrm{KC}$, and with or without treatment before LT differed. Although our institution is the largest LT center in Japan, a multicenter, nationwide study is needed to confirm the present findings. Among a considerable number of patients who were registered in this study between January 1999 and August 2015, 109 of 159 patients had been treated with TACE, PEIT, RFA and/or HR before undergoing LT. In fact, various diagnostic methods, treatment modalities, surgical techniques or perioperative management strategies for HCC recurrence emerged and became significantly improved during this period. Therefore, patients who were treated with these modalities over several years should be compared to exclude bias. The present findings were generated from a single-center study. A nation-wide study is needed to confirm the present findings.

\section{Conclusions}

Outcomes after LT are favorable for patients with small HCC that meet the MC, or are less biologically malignant. 


\section{Acknowledgements}

None.

\section{Footnote}

Conflicts of Interest: The authors have no conflicts of interest to declare.

Ethical Statement: The Ethics Committee of Kyoto University approved the study, which proceeded in accordance with the Declaration of Helsinki, 1996.

\section{References}

1. Global battle against cancer won't be won with treatment alone--effective prevention measures urgently needed to prevent cancer crisis. Cent Eur J Public Health 2014;22:23, 28.

2. European Association For The Study Of The Liver; European Organisation For Research And Treatment Of Cancer. EASL-EORTC clinical practice guidelines: management of hepatocellular carcinoma. J Hepatol 2012;56:908-43.

3. Starzl TE, Iwatsuki S, Van Thiel DH, et al. Evolution of liver transplantation. Hepatology 1982;2:614-36.

4. Kokudo N, Makuuchi M. Evidence-based clinical practice guidelines for hepatocellular carcinoma in Japan: the J-HCC guidelines. J Gastroenterol 2009;44 Suppl 19:119-21.

5. Yao FY, Xiao L, Bass NM, et al. Liver transplantation for hepatocellular carcinoma: validation of the UCSFexpanded criteria based on preoperative imaging. Am J Transplant 2007;7:2587-96.

6. Mazzaferro V, Llovet JM, Miceli R, et al. Predicting survival after liver transplantation in patients with hepatocellular carcinoma beyond the Milan criteria: a retrospective, exploratory analysis. Lancet Oncol 2009;10:35-43.

7. Sugawara Y, Tamura S, Makuuchi M. Living donor liver transplantation for hepatocellular carcinoma: Tokyo University series. Dig Dis 2007;25:310-2.

8. Toso C, Asthana S, Bigam DL, et al. Reassessing selection criteria prior to liver transplantation for hepatocellular carcinoma utilizing the Scientific Registry of Transplant Recipients database. Hepatology 2009;49:832-8.

9. Zheng SS, Xu X, Wu J, et al. Liver transplantation for hepatocellular carcinoma: Hangzhou experiences.
Transplantation 2008;85:1726-32.

10. Takada Y, Ito T, Ueda M, et al. Living donor liver transplantation for patients with HCC exceeding the Milan criteria: a proposal of expanded criteria. Dig Dis 2007;25:299-302.

11. Kaido T, Ogawa K, Mori A, et al. Usefulness of the Kyoto criteria as expanded selection criteria for liver transplantation for hepatocellular carcinoma. Surgery 2013;154:1053-60.

12. Inomata $\mathrm{Y}$, Uemoto S, Asonuma K, et al. Right lobe graft in living donor liver transplantation. Transplantation 2000;69:258-64.

13. Ito T, Kiuchi T, Egawa H, et al. Surgery-related morbidity in living donors of right-lobe liver graft: lessons from the first 200 cases. Transplantation 2003;76:158-63.

14. Morioka D, Egawa H, Kasahara M, et al. Outcomes of adult-to-adult living donor liver transplantation: a single institution's experience with 335 consecutive cases. Ann Surg 2007;245:315-25.

15. Gomaa AI, Khan SA, Leen EL, et al. Diagnosis of hepatocellular carcinoma. World J Gastroenterol 2009;15:1301-14.

16. Yamamoto K, Imamura H, Matsuyama Y, et al. Significance of alpha-fetoprotein and des-gamma-carboxy prothrombin in patients with hepatocellular carcinoma undergoing hepatectomy. Ann Surg Oncol 2009;16:2795-804.

17. Miyaaki H, Nakashima O, Kurogi M, et al. Lens culinaris agglutinin-reactive alpha-fetoprotein and protein induced by vitamin $\mathrm{K}$ absence II are potential indicators of a poor prognosis: a histopathological study of surgically resected hepatocellular carcinoma. J Gastroenterol 2007;42:962-8.

18. Kobayashi M, Ikeda K, Kawamura Y, et al. High serum des-gamma-carboxy prothrombin level predicts poor prognosis after radiofrequency ablation of hepatocellular carcinoma. Cancer 2009;115:571-80.

19. Kurokawa T, Yamazaki S, Mitsuka Y, et al. Prediction of vascular invasion in hepatocellular carcinoma by nextgeneration des-r-carboxy prothrombin. Br J Cancer 2016;114:53-8.

20. Inomata $\mathrm{Y}$, Tanaka $\mathrm{K}$, Egawa $\mathrm{H}$, et al. The evolution of immunosuppression with FK506 in pediatric living-related liver transplantation. Transplantation 1996;61:247-52.

21. Takada Y, Ueda M, Ito T, et al. Living donor liver transplantation as a second-line therapeutic strategy for patients with hepatocellular carcinoma. Liver Transpl 2006;12:912-9.

22. Fukumitsu K, Hammad A, Kaido T, et al. Validation 
of steroid-free immunosuppression regimen after liver transplantation. J Clin Gastroenterol Treat 2015;1:2.

23. Fisher RA, Kulik LM, Freise CE, et al. Hepatocellular carcinoma recurrence and death following living and deceased donor liver transplantation. Am J Transplant 2007;7:1601-8.

24. Lo CM, Fan ST, Liu CL, et al. Living donor versus

Cite this article as: Kamo N, Kaido T, Yagi S, Okajima H, Uemoto S. Liver transplantation for small hepatocellular carcinoma. HepatoBiliary Surg Nutr 2016;5(5):391-398. doi: 10.21037/hbsn.2016.05.03 deceased donor liver transplantation for early irresectable hepatocellular carcinoma. Br J Surg 2007;94:78-86.

25. Ninomiya M, Harada N, Shiotani S, et al. Hepatocyte growth factor and transforming growth factor beta 1 contribute to regeneration of small-for-size liver graft immediately after transplantation. Transpl Int 2003;16:814-9. 